\title{
High accuracy three-dimensional shape measurements for supporting manufacturing industries
}

\author{
- Establishment of the traceability system and standardization -
}

\author{
Sonko Osawa*, Toshiyuki Takatsuji and Osamu Sato
}

[Translation from Synthesiology, Vol.2, No.2, p.101-112 (2009)]

\begin{abstract}
Digital engineering technique has widely been used for speedy and effective manufacturing of industrial products. Coordinate measuring machines (CMM) are being used for measuring three-dimensional shapes of products and taking an important role to keep the quality of the products high. National Metrology Institute of Japan (NMIJ/AIST) has established the traceability system of CMM, that is, measurement results by CMM are traceable to the national standards of length. To achieve this objective, not only calibration techniques have been developed, but industrial standards (ISO and JIS) and guidelines to which accredited laboratories should conform were documented. In addition, to raise the calibration capabilities of prefectural laboratories and to contribute to the local industries, technical supports have been provided by NMIJ.
\end{abstract}

Keywords : Three-dimensional coordinate measurement, coordinate measuring machine, measurement standard, metrological traceability, digital engineering

\section{Introduction}

Japan became a wealthy country by developing its industry after World War II. Particularly, manufacturing, represented by the automobile industry, is the center of Japanese economy. In manufacturing, a product is produced by designing, manufacturing, and assessment. Recently, manufacturing is done using digital data for design, manufacturing, and assessments as shown in Fig. 1. After designing using CAD (computer aided design) and CAE (computer aided engineering), the data is sent to CAM (computer aided manufacturing) for manufacturing. The manufactured product is measured using CAT (computer aided testing), and the measurement data are fed back to the design and manufacturing processes. The analysis and design based on real data as well as the manufacturing process are improved, and this completes the cycle of the manufacturing process. Through the development of the manufacturing system using digital data, it is possible to significantly reduce the days required for manufacturing prototypes. In such digital manufacturing system, coordinate measuring machines (CMM) are often used to assess the shape of the product. CMM is also called the universal measuring machine, and is capable of measuring various three-dimensional shapes (position, size, and geometrical features including straightness, roundness, cylindricity, and squareness).

Here, the functions of a CMM will be outlined. As shown in Fig. 2, the CMM consists of a probing system to measure the coordinates by contacting the surface of the workpiece, a guide to linearly transfer the probing system along the $\mathrm{X}$,
$\mathrm{Y}$, and $\mathrm{Z}$ axes that run at right angles to each other, a scale to measure the distance of transfer, and a computer to control the coordinate transformation and the measuring machine. In the CMM, the range in which the probe (stylus) attached to the probing system can move is its measurement space. It is also possible to calculate not only the distance between two points, but also the feature quantity of geometrical shape of the workpiece (for example, diameter, roundness, and center coordinate of a circle) using the least-square fitting from measured points.

Conventionally, CMMs were expensive and required advanced skills to operate, and they were installed only in prototype and quality control divisions of large corporations. However, they are currently installed in manufacturing lines and small factories because of the need to adapt to digital manufacturing, demand for high quality control for products, and for differentiation against products of other Asian countries. By installing the workpiece and building

Circulation of data in digital engineering

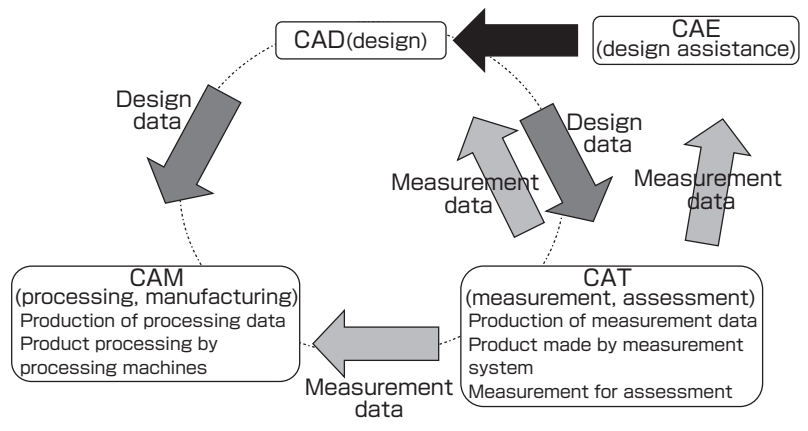

Fig. 1 Digital data manufacturing.

National Metrology Institute of Japan, AIST Tsukuba Central 3, Umezono 1-1-1, Tsukuba 305-8563, Japan * E-mail : sonko. osawa@aist.go.jp

Received original manuscript December 12, 2008, Revisions received January 27, 2009, Accepted May 11, 2009 
the measurement program on the first run, the measurements thereafter on the CMM can be done by computer control. Therefore, some companies simply assign measurement personnel to only conduct routine work, and depend on the manufacturer of the measuring machine to do the complex programming to cut the cost of measurements. However, it is known that there is a distinct difference between the measurements by a person with specialized knowledge of $\mathrm{CMM}$ and someone without such knowledge. This is because the difference in the knowledge and the ability to deal with factors such as positioning of the workpiece and temperature of the environment may affect measurements. To increase the reliability of three-dimensional measurement, it is most important to create a system where the skill of the operator can be improved without requiring profuse knowledge or prolonged training.

Previously in Japan, the manufacturing process was perfected within the consolidated company group, and the system for maintaining reliability of the product was relatively well established within the companies. However, as more less-expensive, good-quality parts are obtained inside and outside Japan, and as production overseas is increased to cut the cost of labor, globalization of parts procurement increases and the system for maintaining reliability within a corporate group is collapsing. For example, flaws in assembly may become a problem because the size of parts delivered by Company A and the size of parts from Company B are slightly different. Therefore, some kind of official support has become necessary to replace the system of maintaining reliability that had been established within the consolidated company group.

The aim of this research is to develop technology necessary to increase reliability of three-dimensional shape measurement, which is one of the most basic technologies that support manufacturing, and to create an official system to diffuse such system to the sites of production.

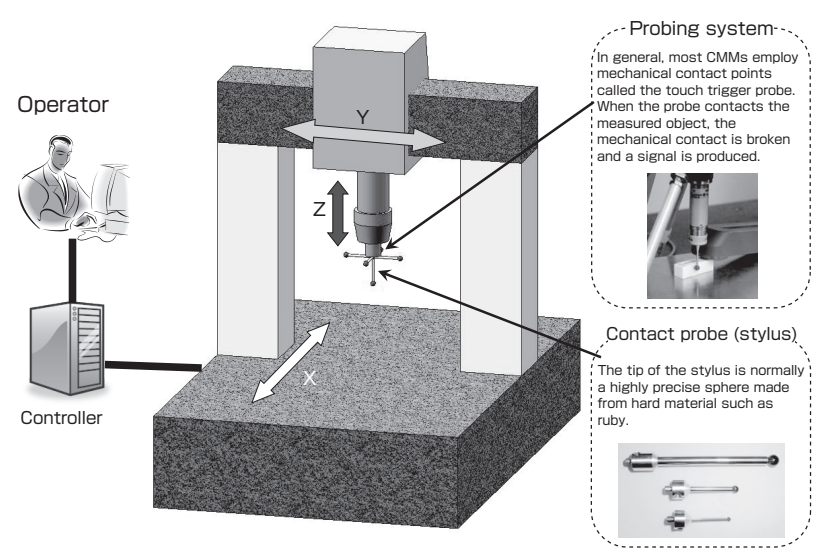

Fig. 2 Outline of CMM.

\section{Scenario}

\subsection{Scenario for improving reliability}

For the dissemination of AIST's research results to a wide range of industries, it is necessary to create a system for delivering the results to the site of production. A scenario for wide dissemination to sites of production was drafted in advance, starting with the establishment of a high-precision national measurement standard. Figure 3 shows the scenario drafted to improve the reliability of three-dimensional shape measurement.

To organize the national standard, AIST develops the standards and their calibration methods, and also will engage in the development of next-generation standards with higher precision. The reliability of three-dimensional measurement is improved by using such developments to calibrate the CMMs at the site of production. The equivalency of the established national standard will be checked by conducting international comparisons that involve the comparison of measured values with the national metrology laboratories of other countries, and the consistency of measured values based on that standard will be recognized worldwide. To diffuse the established standard in Japan, an accreditation system of calibration service is constructed, and the standard is introduced into the site of production through the service provided by the calibration service. To increase the competitiveness of local small and medium companies, training and technical support for highprecision measurement are provided to public research laboratories of each prefecture. Also, the reliability of threedimensional shape measurement is raised at the site of production by standardizing the CMM assessment methods and the new three-dimensional shape measurement methods, and by developing a remote calibration technology to allow calibration of the CMM on-site using a simple procedure. To support Japanese companies that operate in developing countries, technical support is provided to national metrology laboratories of the developing countries. By establishing the scenario, our objective was to improve the international competitiveness of the Japanese manufacturing industry.

\subsection{Maintenance of traceability and goals of development}

Objective demonstration of the reliability of threedimensional shape measurement can be achieved by establishing the traceability to the national standards. For over ten years, AIST has been engaging in technological development to establish the traceability system of threedimensional measurement. Figure 4 shows the technological developments of the traceability system for length. For threedimensional measurement, the system is traceable to the iodine stabilized He-Ne laser that is the national standard for length. The shape of the product manufactured is assessed by a CMM; this CMM is calibrated with a standard called 
gaugeblock or ball plate, which is calibrated with a laser distance meter using stabilized He-Ne laser; and this laser distance meter is calibrated with the iodine stabilized HeNe laser that is the national standard of length. Hence, the standards are linked seamlessly to the higher-level standards.

For practical operation of such traceability system at the sites of production, the following four items must be newly developed: (1) a calibration system of the standard, (2) an accreditation system for private calibration services, (3) standardization of CMM assessment method using standards, and (4) a training system to improve the skills of measurement personnel. To establish the traceability system for three-dimensional measurement, Full Research was conducted with the objective of developing these four subsystems. The researches conducted by AIST to develop these subsystems included the following three research topics:

1. Development of standards to calibrate and assess the CMM (construction of metrology standard)

2. Technological development for constructing the traceability system for three-dimensional measurement (construction of a calibration service accreditation system and standardization of calibration methods)

3. Development of high-precision three-dimensional shape measurement technology (advancement of metrological technology)

All three are developments that improve the reliability of three-dimensional shape measurement required in manufacturing, and are basic technologies to maintain reliability of measurements at the site of production. In chapters 3 to 5 , specifics of the above technological developments will be described. Chapter 6 will be a description of the activities currently conducted at AIST for three-dimensional shape measurement technology, activities to diffuse the metrological standards to sites of production, and future developments.

\section{Standard for assessing CMM}

\subsection{Error in three-dimensional measurement}

Although CMM is a convenient and useful measuring device, there are the following issues concerning its reliability:

1. Measurement error is likely to occur because the probe that detects the position of the workpiece does not match

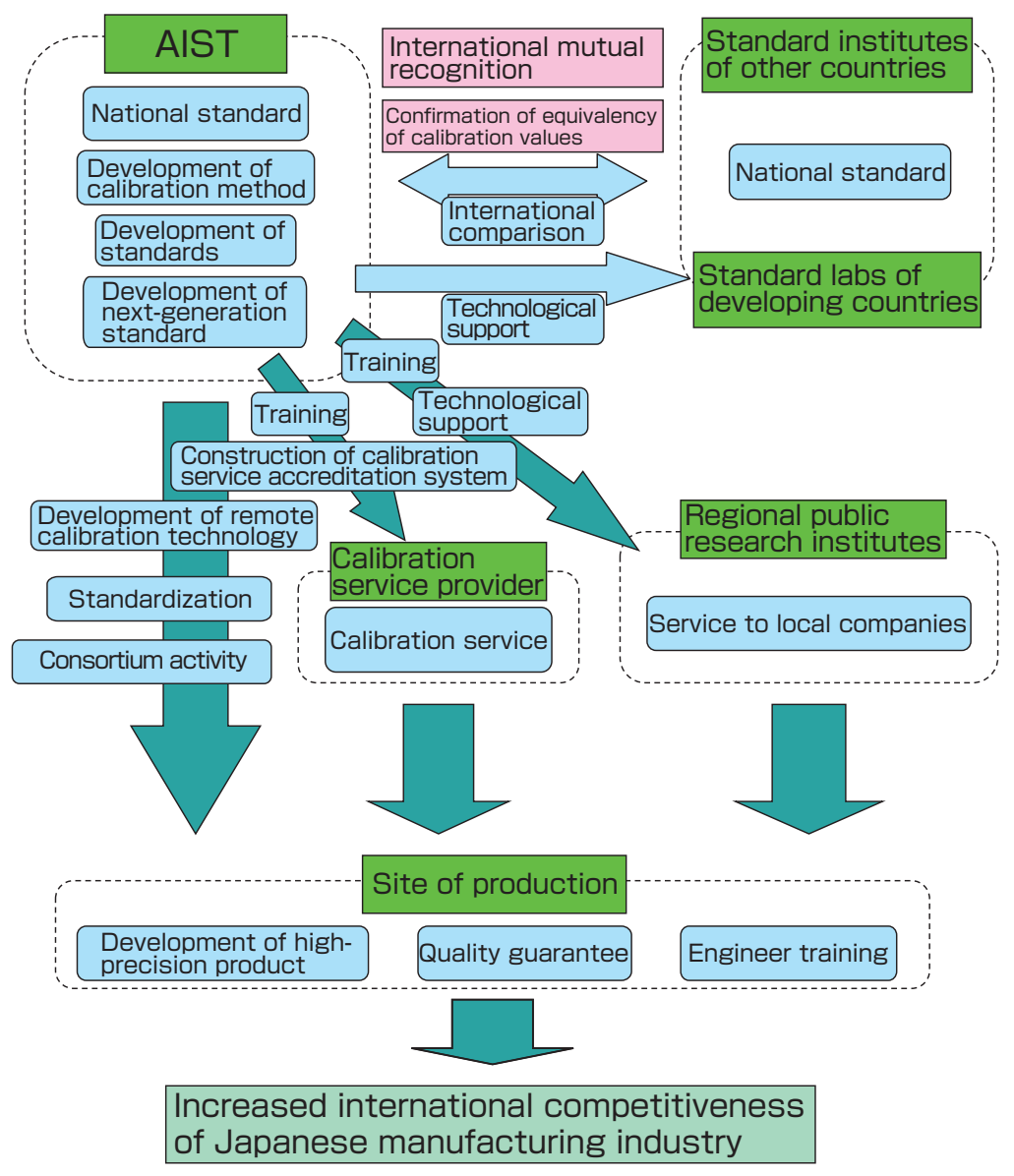

Fig. 3 Scenario for improving reliability of three-dimensional shape measurement. 
the baseline length of the scale (that is, position of the probe is separated from the scale itself) (in technological terms, it "does not fulfill Abbe's Principle").

2. There are several other factors of error, and assessment of uncertainty of measurement data is difficult.

Although Issue 1 is a major problem in conducting highprecision measurement, the effect is kept small by allowing correction by software ${ }^{[1]}$, by improving repeatability through increased rigidity of the machine itself. Most of the current CMMs have software correction functions, and it is necessary to obtain accurate correction data in advance to conduct effective correction. Specifically, there are two types of correction data. One is the correction data for the probing system. By measuring a calibration sphere, for which the value of the diameter has been precisely measured in advance and which has extremely small shape error (with $50 \mathrm{~nm}$ or less deviation from circularity), the diameter, deflection, and characteristic of this probing system of the spherical tip of the probe (stylus) used can be calculated (specifically, when the cross-section of the sphere is measured with a CMM, the shape may not turn out circular, but may be triangular or square depending on the characteristic of the probing system). The other type of correction data is the movement error of the instrument including scale error (of attachment of the scale), squareness error (orthogonality among each axis), straightness error (distortion in each axis guide), and rotational error (error due to changes in position). The errors of scale, squareness, straightness, and rotation are called geometrical errors ${ }^{[2]}$ that can be calculated using various standards. Standards for CMM are necessary to obtain precise measurements of the two types of correction data, as described in the next section.

Issue 2 refers to the difficulty in assessment of uncertainty because CMM has multiple factors of error, and also because of complicated processing where the measurement data is calculated by concentrating the discretely distributed

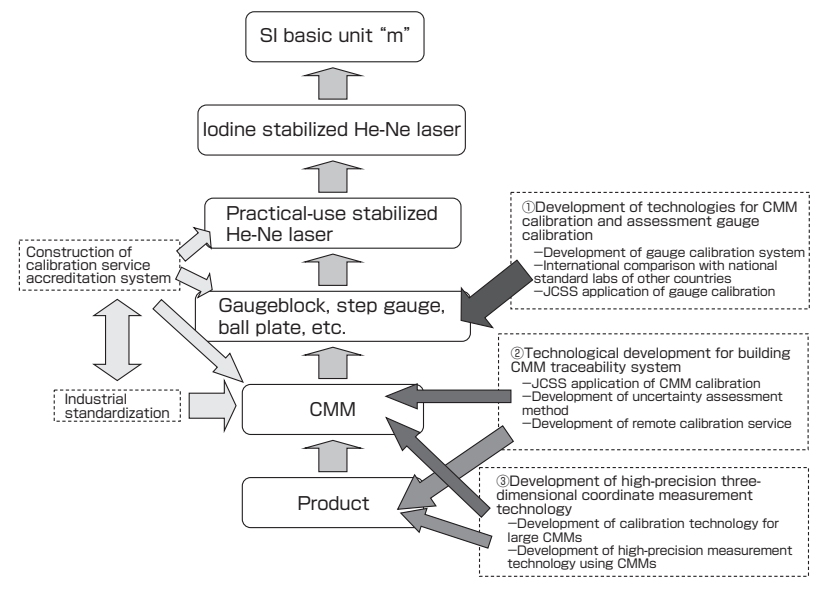

Fig. 4 Relationship between traceability system and Full Research. measurement points into one factor. We looked at a new calculation method for uncertainty using software simulation, and conducted research to solve this problem. This will be explained in detail in section 4.2.

\subsection{Development of standards for CMM \\ 3.2.1 Step gauge}

To check the measurement accuracy of the CMM or to obtain the data for software correction, various standards such as gaugeblocks and ball plates that can be traced to higher-level national standards (iodine stabilized $\mathrm{He}-\mathrm{Ne}$ laser) are used. Unless calibration of these standards is conducted accurately, highly precise assessment of CMMs that is in the lower level of the traceability system cannot be done. Therefore, development of standard calibration technology is important, and the metrology labs of other countries are engaging in the development of calibration technology and calibration services.

AIST has been engaging in the development of a standard calibration system for about 10 years. In Japan, end measures (gaugeblock or step gauge) are generally used in the precision assessment of CMMs. For assessment of CMM, a step gauge (see Fig. 5), where short gaugeblocks are lined up and used as standards for varying lengths, is used more often than the gaugeblock. In major national metrology labs, step gauges are calibrated using a special instrument that combines a laser interferometer and a moving stage ${ }^{[3]}$. AIST developed a system for calibrating the step gauge by combining the CMM and the laser interferometer ${ }^{[4]}$. Figure 6 shows the developed system. In this system, by using a four optical path interferometer, the measured length shows the distance between the center of the sphere at the tip of the stylus and the interferometer at all times, even if rotation errors such at pitching and yawing occur in the stylus. When the step gauge with measured length $500 \mathrm{~mm}$ was calibrated using this system, uncertainty of $0.30 \mu \mathrm{m}$ (95\% confidence interval) was achieved.

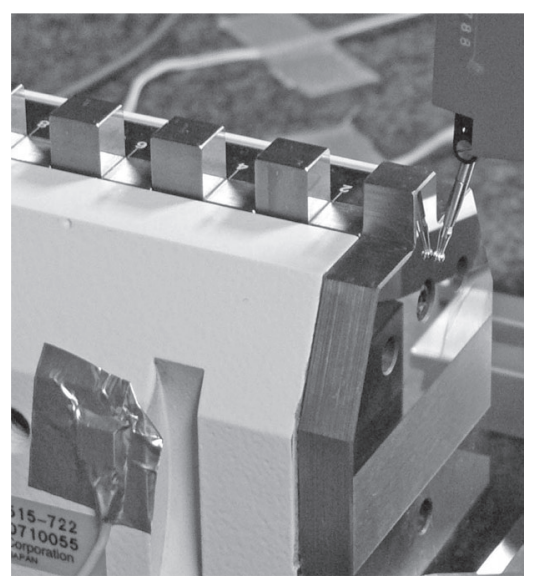

Fig. 5 Exterior view of step gauge (measurement surface is arranged in comb-tooth form). 


\subsubsection{Ball plate}

In Europe, a ball plate and a hole plate (see Fig. 7) that allow two-dimensional assessments are used more often for assessment and calibration of CMM than end measures that are one-dimensional standards. The central coordinates of a sphere or a cylinder placed in the standards are determined, and the CMM assessment is conducted using these coordinates. Compared to end measures, it is possible to obtain more information and know precise errors within the measurement rage. To make the two-dimensional standards available in Japan, AIST constructed a calibration system for two-dimensional standards ${ }^{[5]}$.

Here, the calibration system of a ball plate will be outlined. A ball plate cannot be calibrated using laser distance meters due to its round shape. Therefore, calibration is done using a $\mathrm{CMM}$, but it is impossible to calibrate beyond the accuracy of the CMM when the results of the CMM measurement are used directly. Therefore, central coordinates of each sphere are measured using a method called the inverse method to reduce the geometrical error of the CMM. In measurements using the inverse method, the error of the scale itself (normally, primary tilt factor) remains, and this is corrected using a standard that is traceable to the length standard to calculate the final scale error. Normally, gaugeblocks with differing lengths are used for this correction, but a laser distance meter was used at AIST. Therefore, extremely precise correction data was obtained. By using this system,

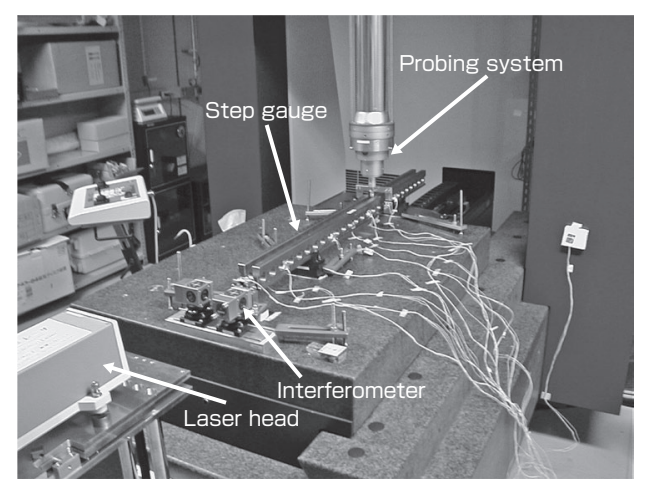

Fig. 6 Step gauge calibration system.

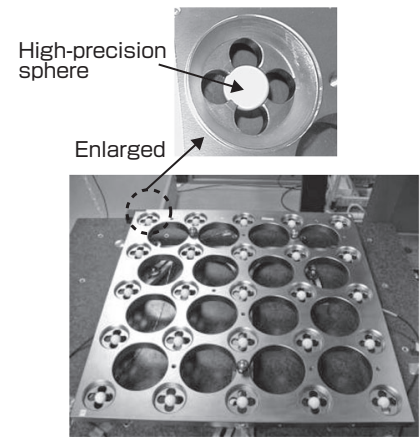

(a)Ball plate

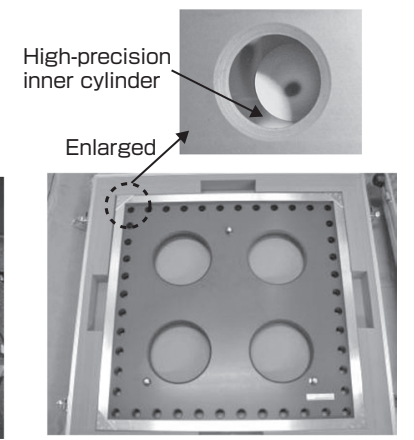

(b) Hole plate
Fig. 7 Two-dimensional standard. uncertainty $0.37 \mu \mathrm{m}$ (95\% confidence interval) was achieved when calibrating the ball plate with measurement length $500 \mathrm{~mm}$. Figure 8 shows the calibration of a ball plate using a laser interferometer. Currently, there are only five laboratories including AIST in the world that use advanced laser interferometer technology for ball plate calibration.

AIST also developed a new standard that can be traced to length standards to enable high-precision calibration of a ball plate at laboratories that do not possess an interferometer ${ }^{[6]}$. This standard is called the ball step gauge, and is in the form of one-dimensionally arranged balls. The sphere which will be measured is positioned at the neutral axis in the crosssectional secondary moment of the $\mathrm{H}$-shape main plate, and it is designed to keep the changes in relative positions to extremely small levels even when positional changes occur due to distortion by the sphere's own weight or heat distortion in vertical and horizontal directions. The distance between the spheres is calibrated at AIST using the laser distance meter, and then supplied to the user. To check the efficacy of this standard, and as part of the activities of the Council of Promotion of Industrial Technology Collaboration, comparative measurements of a ball plate were conducted by circulating this standard among regional public research laboratories. As a result, almost all labs agreed at a deviation within $0.5 \mu \mathrm{m}$ against the AIST calibration value. It was confirmed that this standard was effective for comparative measurement, and that the technological level of the regional public laboratories were sufficiently high ${ }^{[5]}$.

\subsection{International comparison of standards 3.3.1 International comparison of step gauges}

The international comparison (CCL-K5) for calibration of step gauges was conducted from 1999 to 2002, with the participation of major national metrology institutes. The pilot laboratory prepared a step gauge with excellent stability, this gauge was transported to the metrology institutes of various countries to conduct blind measurements, and the results were reported to the pilot laboratory. The nine national

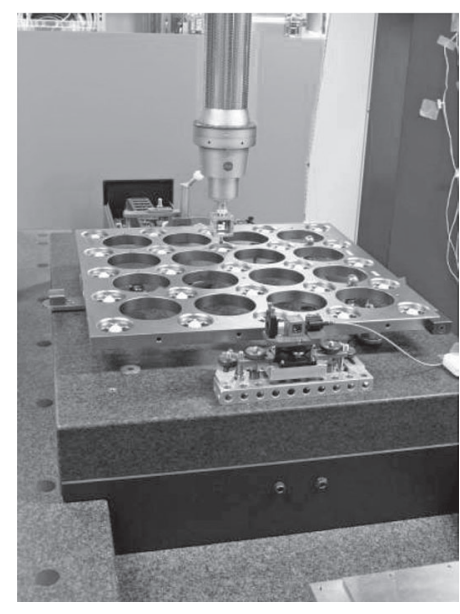

Fig. 8 Ball plate calibration. 
metrology institues participated with this comparison.

Figure 9 shows the results of the international comparison ${ }^{[7]}$. In the international comparison, the participating institutes conduct measurements independently based on the national standard of their respective countries, and therefore none of the labs know which of their results are the closest to the true value. The weighted average value considering the uncertainties of measurements of the participating labs is designated as a value that is probably the most certain (reference value), and the deviation of the data of each lab is calculated. However, in this international comparison, it was found that the measurement data of some of the participating labs were greatly deviant. Ultimately, the measurements of four countries, Japan (NMIJ/AIST), U.S.A. (NIST), Switzerland (METAS), and Germany (PTB) were in good agreement, and therefore the average values of these four laboratories were used as a reference value. The graph of Fig. 9 is a plot showing the deviation from the reference value for each lab (the values of AIST run only to $720 \mathrm{~mm}$ due to the limitations of the measurement system). From this result, the reliability of the step gauge calibration system developed at AIST was confirmed, and the high level of the calibration technology of AIST was presented to the world.

\subsubsection{International comparison of ball plates}

The international comparison of ball plate calibration (CCL-K6) was conducted from 2001 to 2004. There were 12 participating institutes. Figure 10 shows the result of this international comparison. The plotted points in this graph show the difference between the AIST result and the reference value, which is the average value of all participating institutes for the distances from 1st sphere to each sphere (2nd to 25th). The number of spheres is shown below the error bar. The error bar shows the uncertainly (66 $\%$ confidence interval) for measurement values of AIST, and the blue line shows the uncertainty (66\% confidence interval) against the reference value. It can be seen from this graph that the AIST values and the reference value match within the range of uncertainty. From this result, the reliability of the developed system was confirmed and the high-level calibration technology of AIST was presented to the world.

\subsection{Calibration service of standards by private companies}

The mission of AIST is to have the technology confirmed in the above international comparison be used widely in industry. Since the number of calibration services that can be undertaken by AIST is limited, private companies with high-level calibration technology can conduct the service and participate as the middle tier of the traceability system, and diffuse the highly reliable three-dimensional shape measurement technology to industry. The Japan Calibration Service System (JCSS) based on the Measurement Act is a mechanism for officially accrediting private companies with proper calibration capacity. The service provider accredited under this system can issue calibration certificates that certify that a standard is traceable to the Japanese national standard. To establish a calibration system for step gauges using this system, a technological committee was established in the National Institute of Technology and Evaluation (NITE), and a draft of Guidelines for Technological Requirements ${ }^{[8]}$ needed for accredited service providers was drafted under the leadership of AIST. Immediately after the establishment of the system, several service providers were accredited, and the traceable step gauges are now employed in several industries.

\section{Reliability assessment of the CMM}

\subsection{Accreditation of calibration service providers for CMM}

As mentioned in chapter 3, it became possible to calibrate the CMM appropriately since the standard for step gauges was established. In this situation, the service providers that

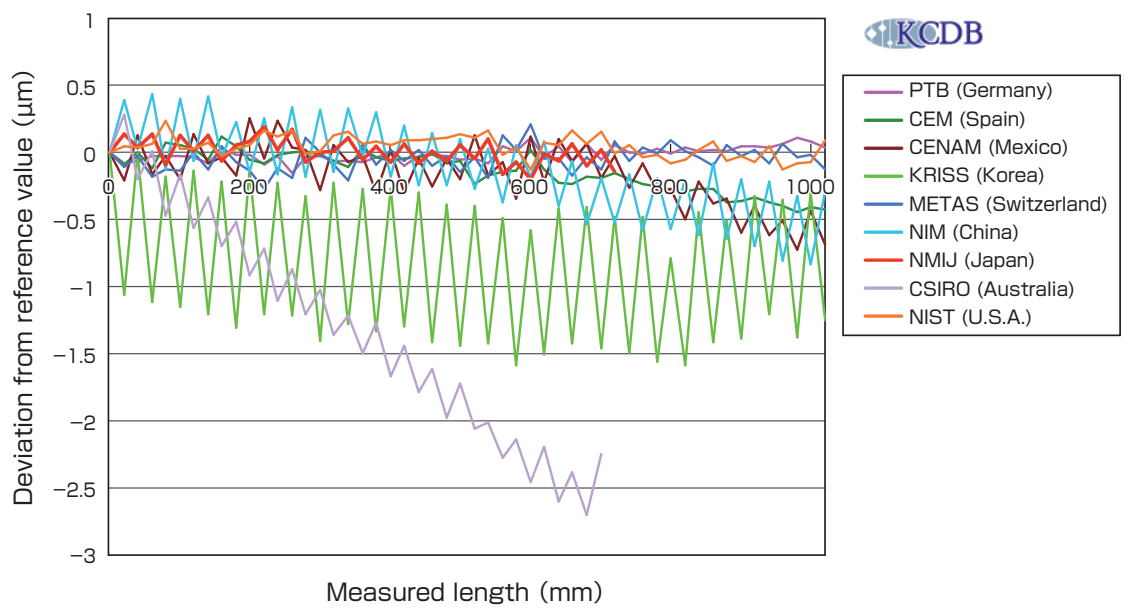

Fig. 9 Result of international comparison for step gauge (CCL-K5). 
own the CMMs requested official certification for their capability to conduct three-dimensional measurements traceable to the national standard. Therefore, similar to the aforementioned step gauge standards, we established a technological committee for officially accrediting the service providers for three-dimensional measurement in the National Institute of Technology and Evaluation (NITE), and drafted the Guidelines for Technological Requirements ${ }^{[9]}$ for CMM calibration. Currently several companies received the official accreditation for CMMs and are providing calibration services.

Since CMM is a multifunctional device, it is impossible to sufficiently evaluate all of its functions just by a few measurements with step gauges. The challenge is to conduct appropriate assessment with as little procedures as possible. The same problem arises in the performance testing for the buyer and the seller of CMM. In ISO, the testing method at the time of delivery of CMM is standardized. AIST has participated in the ISO meetings as an expert to work on standardization. In determining the Guidelines for Technological Requirements for the calibration of CMM, we decided to use the ISO standards ${ }^{[10]}$. This means that the metrological standard (metrological traceability system) references the industrial standard (standardization and rules for products and services). On the other hand, this ISO standard rules that a standard traceable to national standard must be used, and in reverse, the industrial standard references the metrological standard. We have been conducting R\&D under the thinking that effective application of the system is possible for use in industry through unification of metrological and industrial standards. This time, one of our efforts was rewarded.

\subsection{Calculation of uncertainty in three-dimensional measurement}

CMM conducts point measurements of each point and

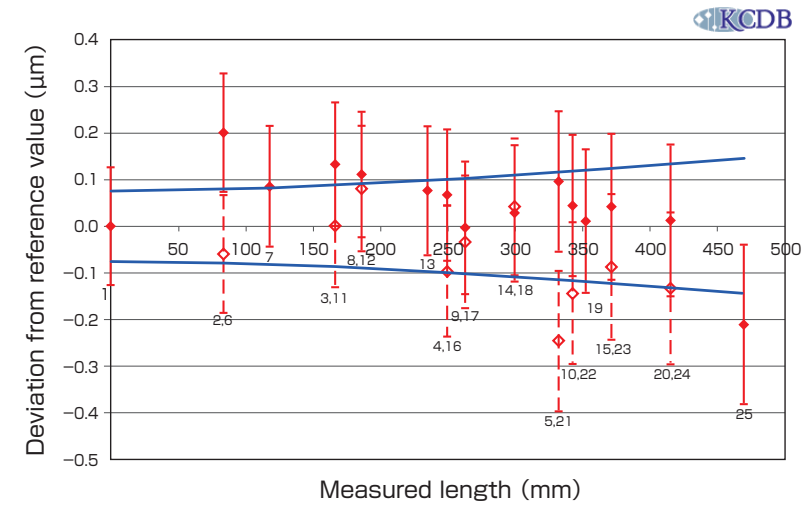

Fig. 10 Result of international comparison for ball plate (CCL-K6).

The graph shows the difference between AIST values and reference values for 25 spheres of the ball plate. The error bar shows the uncertainty of AIST value, while the blue line shows the uncertainty of reference value. gathers the point data. If the measurement is for a circle, least-square fitting is done to calculate the diameter, circularity, and central coordinates. Since there are measurement errors in each measurement point, it is difficult to see at which uncertainty the diameter and circularity were finally calculated. Following items can be listed as factors of uncertainty in three-dimensional measurement (see Fig. 11).

1. Uncertainty of probing

2. Uncertainty arising from geometrical error

3. Uncertainty of data processing (least-square method, etc.)

4. Uncertainty arising from measurement procedure (number of measurement points and their arrangement, etc.)

5. Uncertainty arising from environmental change (temperature, humidity, etc.)

6. Uncertainty arising from positioning of workpiece (holding strength, distortion from own weight, etc.)

7. Uncertainty arising from workpiece itself (surface roughness, shape error, etc.)

Since various factors of uncertainty affect the measurement, it is fairly complicated to assess the final uncertainty. At AIST, with a grant from the International Joint Research Program, New Energy and Industrial Technology Development Organization (the NEDO grant), research on measurement uncertainty in CMM using Monte Carlo simulation was conducted jointly with the PhysikalischTechnische Bundesanstalt (PTB) of Germany, the National Measurement Institute of Australia (NMIA), the University of Tokyo, and others. The method is called virtual $\mathrm{CMM}^{[1]}$, and its basic concept was developed by the PTB. Figure 12 shows the outline of the virtual CMM. Virtual CMM is a computer model of CMM including the errors of factors of uncertainly such as geometrical errors. By conducting about 200 measurements on the virtual model on the computer using the measured position information and other data obtained from actual measurements, the standard deviation and the uncertainty of the measured value obtained from virtual measurements are calculated.

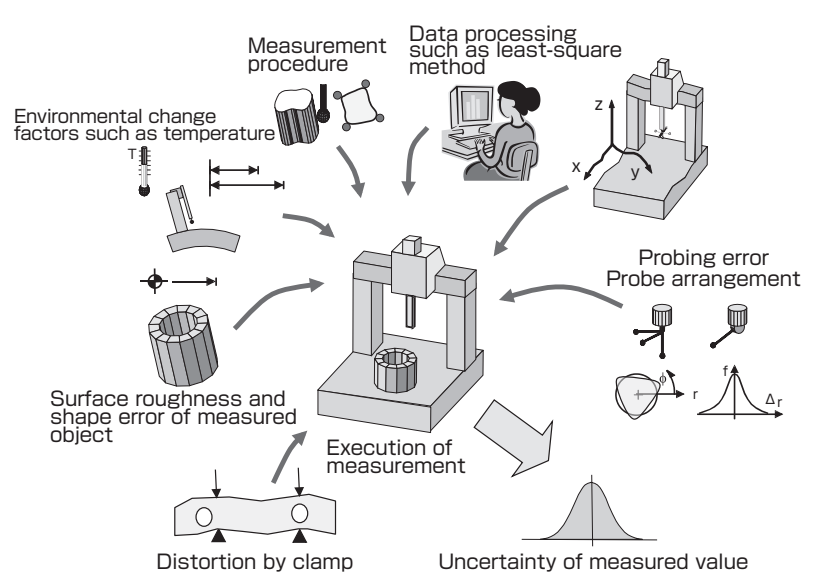

Fig. 11 Factors of measurement uncertainty for СMM. 
In this study, AIST conducted comparative measurement of a normally workpiece, and assessed the practical usability of the virtual CMM. The diffusion of this virtual CMM technology helped the construction of a traceability system for three-dimensional measurement. The calculation method of uncertainty by simulation was standardized as ISO/TS 15530-4.

\subsection{Remote calibration of CMM}

To calculate the uncertainty using the method mentioned in the previous section, it is necessary to obtain data for geometrical error for the entire measurement space using standards. Since extremely specialized technique is needed to obtain the geometrical error data of measurement space of the CMM, this measurement is preferably done by a specialized calibration service. However, time and money costs are needed when the calibration specialist is dispatched to the site of production. Therefore, AIST developed a system to conduct this work easily using the Internet ${ }^{[12]}$.

Figure 13 shows the outline of remote calibration of CMM using the Internet. The calibration service provider first sends a standard to the CMM user. Changes during transportation such as temperature, humidity, and vibrations are monitored by an accompanying sensor with a recording function. The calibration service determines whether the standard has changed by looking at the recorded information of the sensor. Next, the user's CMM is calibrated using a twodimensional standard. The standard is set in position by the user, while the setting procedure is monitored by a specialist of the calibration service using the network camera. The user's CMM is controlled by the calibration specialist via the Internet. The environmental temperature during calibration is measured using the thermometer delivered along with the standard. The temperature measurement data are also sent using the Internet and are stored by the calibration service. The parameter setting and the creation of a coordinate system of the probing system are done by talking directly with the user via telephone or Internet camera phone. After completing the measurement, the calibration service uses these measurement data to calculate the geometrical error of the user's CMM. The calculated geometrical error is sent to the user, and used for calculating uncertainty and

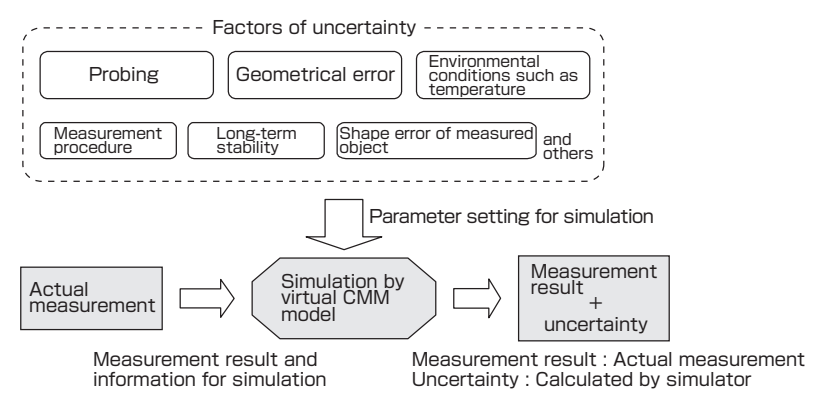

Fig. 12 Outline of virtual CMM. error corrections. The calibration service specialist does not have to be present at the site of calibration, and we call this remote calibration. This technological development enables traceability of three-dimensional measurement without requiring the user to have knowledge in standards and geometrical error calculation, and can be done at low cost.

AIST has been conducting CMM calibration service using the remote method as a requested test since 2005. This service is conducted by the assessment method based on ISO standards using gaugeblocks and step gauges rather than two-dimensional standards, just as in the CMM calibration in the JCSS.

\section{Sophistication of three-dimensional shape measurement}

\subsection{Calibration of large CMMs}

For shape measurement of automobile body or aircraft fuselage, a large CMM with measurement space such as $5 \mathrm{~m}$ $\times 3 \mathrm{~m} \times 2 \mathrm{~m}$ is used. For calibration of such large CMM, large standards may be used, although there are issues of weight and time cost. Therefore, AIST suggested a calibration system using laser tracking laser interferometer (or laser tracker) as one of the methods to calibrate large CMMs without using a standard. The CMM calibration system using the laser tracker was studied at the National Physical Laboratory (NPL) of U.K., the Physikalisch-Technische Bundesanstalt (PTB) of Germany, and AIST. All devices calculate the coordinates by the principle of trilateration from distance measurement of the device. The devices of NPL ${ }^{[13]}$ and $\mathrm{PTB}^{[14]}$ use repeated measurements of the target position by transferring one laser tracker to several positions, while the AIST method is to install four laser trackers to measure the positions of the target at once ${ }^{[15]}$. Since the AIST method allows calculation of coordinates in one shot, it has merit of having shorter measurement time compared to the NPL and PTB methods. Therefore, the external environment effect such as a shift in coordinates of the workpiece due to changes in temperature can be kept to a minimum.

Figure 14 is a photograph of the laser tracker developed at AIST. The characteristic of this system is to reduce

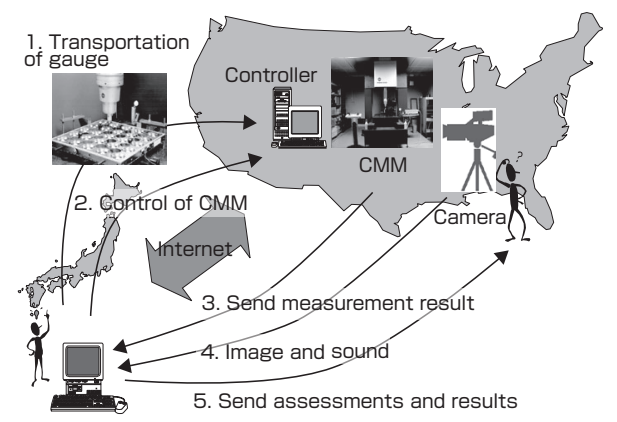

Fig. 13 Remote calibration technology for CMM. 
mechanical error of the tracking system by using a hemisphere for the mirror to scan the laser. Normally, the two axes for scanning the laser in horizontal and vertical directions must be adjusted accurately, and an advanced skill is required for this adjustment. In our device, three spheres are arranged at $120^{\circ}$ intervals, and hemispheric mirrors are fixed to the three spheres to create a high-precision laser scanning mechanism using low-cost mechanical elements ${ }^{[16]}$. Upon comparing this laser tracker with high-precision $\mathrm{CMM}$, it was confirmed that the mechanical precision of the system itself was $0.3 \mu \mathrm{m}$ or less ${ }^{[17]}$. The geometrical error of the CMM was calculated and compared using a ball plate and a laser tracker, and the values matched within $2 \mu \mathrm{m}$ in the measurement space of $300 \mathrm{~mm}$ cube ${ }^{[18]}$.

When the user uses the laser tracker, the ease of handling is important. Therefore, we achieved downsizing and weight reduction by using a spherical motor that was developed by the Intelligent Systems Research Institute, AIST ${ }^{[19]}$. This is a good example of a research result produced by a fusion with other disciplines. With this technological development, highprecision calibrations of large CMM and hand coordinates of industrial robots can be conducted.

Currently, discussions of standardization have been started for the assessment of the laser tracker in ISO meetings, and we plan to contribute to its standardization through our knowledge and experience gained in this technological development.

\subsection{High-precision measurement by CMM}

In industries such as mold making where highly precise manufacturing is done, assessment at higher precision than the innate precision of the measurement machine may be required occasionally. Although normally such assessment is impossible, it is possible to conduct higher precision measurement by special arrangements and procedures that will mutually cancel out the errors of the measuring machine. The inverse method used for calibration of a ball plate is one example. AIST developed such high-precision measurement technology, and we shall present the result for measuring a

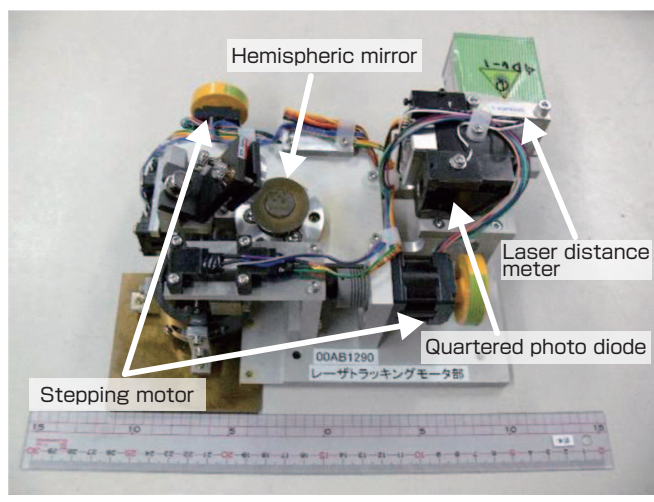

Fig. 14 Laser tracking interferometric distance meter. cylinder as an example.

As shown in Fig. 15, the circularity of a cylinder is measured and assessed by conducting measurement at eight equalinterval points. After the first measurement, the workpiece is rotated 45 degrees for the second measurement. It is again rotated 45 degrees, with measurements for total eight positions. When the average of the eight measurements is calculated, geometrical error, offset error for the two styli, and effects of probe directionalities can be cancelled out. This method is called the multiple measurement method, and can be used for precise measurement of rotationally symmetrical shapes ${ }^{[20]}$. For calibration of standards, AIST is planning high-precision calibration experiments for such applications. This measurement method can be applied in sites of production, and we plan to disseminate this knowledge widely through regional public laboratories.

\section{Future of three-dimensional shape measurement}

\subsection{Measurement standards and standardization for digital engineering ${ }^{[21]}$}

As shown in Fig. 1, in digital engineering where the entire process of design, manufacturing, and assessment is done by digital data, it is necessary to compare the CAD design data and the actual measured results. CAD data has planar information, and the volume of information from discrete measurement data of a conventional CMM with a tactile probing system is insufficient. Therefore, noncontact CMM is now being used, since it can obtain measurements for high-density multiple points in one shot. Previously, for noncontact CMM, the manufacturers were conducting precision assessments and providing precision guarantees using their own standards, and there was no system based on common assessment methods. Hence, there was no uniform index when the user was purchasing a CMM, and it was not possible to determine whether the precision of the instrument was as stated in the catalog. Therefore,

- Measurement point by probe 1

O Measurement point by probe 2
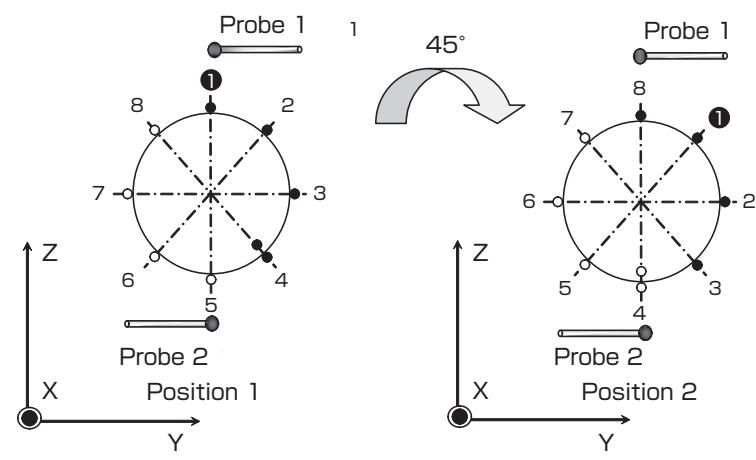

Position 4,5,6,7,8 
AIST established a consortium for creating a standard for the precision assessment of noncontact CMM in 2005. The consortium developed standards for use in the assessment of the noncontact CMM (metrological standards) and devised an assessment method using these standards.

Since there are many different types of noncontact CMMs, it is important to create standards that enable assessment of these various measuring machines. In optical measuring machine, error tends to occur in the presence of luster in the measured area of the standard. Therefore, the surface of the standard must have optically diffusing surface. To select an ideal diffusing surface, through cooperation of the consortium members, several spheres were created with slightly different processing methods, conditions, and surface coatings. Figure 16 shows some examples. Processing were done with over 100 different conditions, the spheres were measured using several types of noncontact CMMs, and a sphere for which relatively stable measurements could be obtained in all measuring machines was selected. Next, using the selected sphere, a ball bar, which would be the standard for a precision assessment of the measuring machines, was created (see Fig. 17). A carbon frame was used so it would not be affected by environmental temperature, since many noncontact CMMs were portable and they were unlikely to be used in a room with constant temperature at $20{ }^{\circ} \mathrm{C}$. Therefore, a standard that was stable against temperature change was needed. Hence, a standard traceable to the national standard was created by the consortium so it could be used for assessment of measuring machines. After conducting comparative measurements, the assessment of noncontact CMM using this standard was standardized as Japan Industrial Standard (JIS). Currently, the drafted JIS is undergoing deliberation at the Japan Industrial Standards Committee as JIS B 7441. It has also been submitted to the ISO committee at the same time. We are collaborating with Digital Human Research Center, AIST, for the standardization of the assessment method of noncontact CMM for the measurement of the human body.

The X-ray CT device that was previously used for the detection of cast pores is now being employed in industry as a CMM that can measure internal structures. Therefore, there are demands from manufacturers and users for the development of a common phantom (standard) to assess
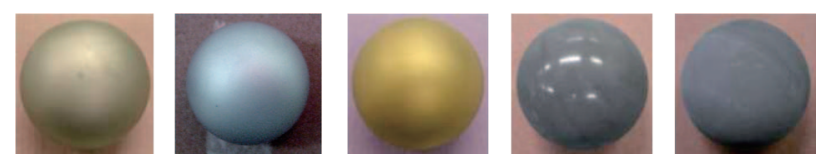

Fig. 16 Spheres manufactured under different conditions.

Dispersion eutectoid coating (B-MOS) (leftmost)

Sandblast: Cr coating (2nd from left), TiN coating (center)

Chemical etching $\left(\mathrm{FeCl}_{2}\right)$ (two on right) the x-ray CT devices, as well as for the standardization of assessment method using the phantom, and AIST is preparing for this study.

\subsection{Cooperation with regional public research laboratories}

Since CMMs are expensive instruments, the financial strain on small to medium businesses is great. Therefore, CMMs are installed in almost all regional public laboratories to provide services, such as requested measurements and the use of the instrument by the local companies. AIST is engaging in activities to improve three-dimensional measurement technology in the Shape Measurement Subcommittee, Intellectual Infrastructure Committee, which is one of the technical committees of the Council of Promotion of Industrial Technology Collaboration. The subcommittee has engaged in comparative measurement of ball plates, demonstration experiment for the calculation of uncertainty in deliberation at the ISO, and assessment experiment of video probe CMM. Also, a project to maintain reliability of CMM measurements is conducted as a local collaborative innovation creation undertaking with public laboratories in the wider Kanto area from FY 2008. Through such activities we are contributing to the improvement of three-dimensional shape measurement technology at the site of production. We hope these technologies will be transferred from public laboratories to local small and medium businesses to vitalize Japanese manufacturing.

\subsection{Operator training}

AIST has engaged in technological developments and diffusion activities for the three-dimensional shape measurement. We feel that the technological development for establishing metrological traceability had been sufficiently organized. In the future, we shall strengthen activities to diffuse daily maintenance methods for CMM to further improve the reliability of the three-dimensional measurement, and train the users. Recent measuring machines including CMMs are digital rather than analog, and the values are shown on the display. Therefore, the operator tends to think that the figures are always correct. However, in measurement, the reliability of the values changes depending

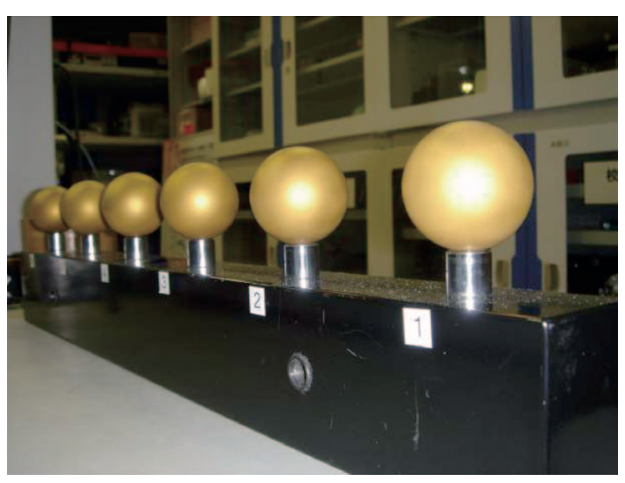

Fig. 17 Ball bar for noncontact CMM assessment. 
on the conditions and methods of measurement. Great difference may occur in measurement reliability depending on whether the operator understands this point or not. For example, some operators may not know that the sizes written on the plan of an industrial product are sizes at $20{ }^{\circ} \mathrm{C}$. To improve the reliability of the product, we believe it is very important to educate the people who are using the assessment tool. We think the training system for improving the skill of the measurement operator is the final requirement for constructing the traceability system at the site of production, and as one step for improving the skills, we plan to create an accreditation system for three-dimensional measurement engineers in the near future.

\section{Conclusion}

Japan, after the World War II, has developed its manufacturing industry in a wide range of fields including automobiles and electronics. CMM is a measuring machine that developed with the increased performance of computers, and plays a major role at the site of advanced manufacturing. Particularly, it is an essential device in the integrated process from digitized design, manufacturing, and assessment. In this paper, we discussed the technological developments related to CMM in manufacturing.

With the goal for strengthening competitiveness of manufacturing, we created a scenario from the establishment of national standards to the diffusion to the site of production, and executed them starting with important items. To this day, the course for three-dimensional measurement using conventional CMMs has been almost entirely organized, except for the accreditation system of operators, but many issues remain to be solved, such as responses to new threedimensional measuring instruments and technologies, as well as the creation of a training system. We plan to create a scenario for new devices and technology, to contribute further to the manufacturing industry.

\section{References}

[1] S. Sartori and G.X. Zhang: Geometric error measurement and compensation of machines, Annals of the CIRP, 44(2), 599-609 (1995).

[2] J.A. Soons, F.C. Theuws and P.H. Schellekens: Modeling the errors of multi-axis machines: A general methodology, Precision Engineering, 14(1), 5-19 (1992).

[3] P.S. Lingard, M.E. Purss, C.M. Sona and E.G. Thwaite: Length-bar and step-gauge calibration using a laser measurement system with a coordinate measuring machine, Annals of the CIRP, 40(1) (1991).

[4] S. Osawa, T. Takatsuji and T. Kurosawa: Development of an interferometric coordinate measuring machine used for step-gauge calibration, Seimitsu Kogaku Kaishi (Journal of the Japan Society for Precision Engineering), 68 (5), 687-691 (2002) (in Japanese).

[5] S. Osawa, T. Takatsuji, T. Kurosawa and K. Umetsu: Zahyo sokuteikiyo nijigen kika geji kosei ni kansuru gijutsu joho (Technological information on calibration of twodimensional geometric gauge for coordinate measuring machine), Sansoken Keiryo Hyojun Monogurafu (AIST Measurement Standard Monograph), 8 (2005) (in Japanese).

[6] S. Osawa, T. Takatsuji, H. Noguchi and T. Kurosawa: Development of a ball step-gauge and an interferometric stepper used for ball-plate calibration, Precision Engineering, 26(2), 214-221 (2002).

[7] CIPM CCL Key Comparison: K5 Final Report.

[8] National Institute of Technology and Evaluation, Bunsho bango JCT20102 gijutsu teki yokyu jiko tekiyo shishin (ichijigen sumpo sokuteiki/burokku geji, kakushu nagasa sokuteiyo koseiki de sokuteimen ga heimen dearu mono (koha kansho sokuteiho ni yoru)) (Document No. JCT20102 Guidelines for Technological Requirements (onedimensional measuring instrument, gaugeblock, calibration instruments for length measurement (by interferometric measurement method))(in Japanese).

[9] National Institute of Technology and Evaluation, Bunsho bango JCT20113 gijutsu teki yokyu jiko tekiyo shishin (zahyo sokuteiki) (Document No. JCT20113 Guidelines for Technological Requirements (coordinate measuring machine)) (in Japanese).

[10] ISO 10360-2:2001 Geometrical product specifications (GPS) - Acceptance and reverification tests for coordinate measuring machines (CMM) - Part 2: CMMs used for measuring size.

[11] K. Takamasu: Virtual coordinate measuring machine, Keisoku To Seigyo (Journal of the Society of Instrument and Control Engineers), 40(11), 801-804 (2001) (in Japanese).

[12] S. Osawa, T. Takatsuji, T. Kurosawa, R. Furuyani and M. Shibata: Traceability system of coordinate measuring machines through Internet, Seimitsu Kogaku Kaishi (Journal of the Japan Society for Precision Engineering), 70(4), 528532 (2004) (in Japanese).

[13] E.B. Hughes, A. Wilson and G. Peggs: Design of a highaccuracy CMM based on multi-lateration techniques, Annals of the CIRP, 49(1), 391-394 (2000).

[14] H. Schwenke, M. Franke and J. Hannaford: Error mapping of CMMs and machine tools by a single tracking interferometer, Annals of the CIRP, 54(1), 475-478 (2005).

[15] T. Takatsuji, Y. Koseki, M. Goto and T. Kurosawa: Restriction for the arrangement of laser tracker in laser trilateration, Measurement Science and Technology, 9(8), 1357-1359 (1998).

[16] Kubifuri undo hikari teko niyoru kosen tsuibi shiki reza kansho sokuchoki tokkyo 3427182 (Tracking laser interferometric distance meter using swinging optical lever, Patent No. 3427182) (in Japanese).

[17] J. Hong, S. Osawa, T. Takatsuji, H. Noguchi and T. Kurosawa: A high-precision laser tracker using an articulating mirror for the calibration of coordinates measuring machine, Optical Engineering, 41(3), 632- 637 (2002).

[18] K. Umetsu, R. Furutnani, S. Osawa, T. Takatsuji and T. Kurosawa: Geometric calibration of a coordinate measuring machine using a laser tracking system, Measurement Science and Technology, 16, 2466-2472 (2005).

[19] T. Yano, T. Takatsuji, S. Osawa, K. Suzuki, Y. Honmura and T. Sakabe: Development of a small two axis spherical motor type laser tracker with submicron measurement accuracy, Denki Gakkai Ronbunshi E (Sensa/Maikuro mashin bumonshi) (IEEJ Trans. SM), 126(4), 144-149 (2006) (in Japanese).

[20] S. Osawa, K. Busch, M. Franke and H. Schwenke: Multiple orientation technique for the calibration of cylindrical workpieces on CMMs, Precision Engineering, 29(1), 56-64 (2005). 
[21] S. Osawa and O. Sato: Hi sesshoku zahyo sokuteiki hyokaho no kogyo hyojunka (Industrial standardization of noncontact coordinate measuring machine assessment method), Keisoku Hyojun to Keiryo Kanri (Measurement Standards and Metrology Management), 57(2), 2-6 (2007) (in Japanese).

\section{Authors}

\section{Sonko Osawa}

Completed doctorate course of the Graduate School of Engineering, Tokyo Denki University in 1998. Doctor of Engineering. Joined the National Research Laboratory of Metrology, Agency of Industrial Science and Technology in 1998. Worked on research of three-dimensional measurement as visiting research of Physikalisch-Technische Bundesanstalt (PTB) of Germany in 2002 2003. To present, works on three-dimensional shape measurement, geometrical quantity measurement, and their standardization. In this paper, worked on overall three-dimensional shape measurement standard.

\section{Toshiyuki Takatsuji}

Completed course in metrological engineering at the Graduate School of Engineering, Kobe University in 1990. Joined the National Research Laboratory of Metrology, Agency of Industrial Science and Technology in 1990. Visiting researcher at the National Metrology Laboratory (NML), Commonwealth Scientific and Industrial Research Organisation (CSIRO) of Australia from 1994 to 1996. Obtained doctor's degree (engineering) in 1999. Currently studies geometrical quantity measurement such as threedimensional measuring machine and planarity. In this paper, integrated the whole research.

\section{Osamu Sato}

Completed doctorate at the Graduate School, The University of Tokyo in 2004. Doctor of Engineering. Joined the National Institute of Advanced Industrial Science and Technology in 2004. Studies size and shape measurement using atomic force microscope, coordinate measuring machine, and coordinate measurement system. Also works on standardization of precision assessment for CMM. In this paper, worked on development of remote calibration technology, measurement standard and industrial standardization for digital engineering, and on establishing collaborative relationship with regional public research institutes. Affiliate of the Japan Society for Precision Engineering.

\section{Discussion with Reviewers}

\section{Overall evaluation}

\section{Comment (Mitsuru Tanaka, Research Coordinator, AIST)}

This paper can be positioned as a Full Research to establish the system for ensuring the reliability of geometrical shape measurement, which is important for promoting Japanese machine parts, for strengthening competitiveness, and for technological transfer. I think it is an excellent research with rich contents.

\section{Answer (Sonko Osawa)}

Measurement is an important ingredient of manufacturing. It determines whether the product is manufactured in accordance to the design plan, and can improve the product value by reflecting the result in the manufacturing process. It is important to maintain the reliability of measurement to increase the value of Japanese products, and I believe the way to achieve this is the traceability system. Creation of this traceability system is a mission of AIST, and I have been engaging in this research by drafting a scenario.

\section{Background of the issue Question and Comment (Mitsuru Tanaka)}

In the past, there was perhaps one coordinate measuring machine at a large manufacturing company. But now, CMMs are installed in small subcontract factories and in each manufacturing line. How about describing the fact that the demand for reliability at the sites of production is increasing, while on the other hand, there is departure from specialized technology to cut cost of the CMM operator?

As background when discussing the contribution of this Full Research to Japanese machine manufacturing businesses, rather than just mentioning "manufacturing," perhaps you should mention the dependency on Europe and US for precision measuring machines, as well as the emerging industrial countries coming up close behind us.

Answer (Sonko Osawa)

I added some descriptions in 1 Introduction.

\section{Explanation of Synthesiology}

\section{Question and Comment (Mitsuru Tanaka)}

While carefully explaining the elemental technologies, it is necessary to discuss how they were synthesized to generate the social outcome. For example, in the relationship with public laboratories, why didn't you take the policy of "diffusing the system in Japan by setting traceability through public labs as the only method"? Why didn't you take the policy of "rather than AIST participating, calibration services can take the technical exam of the International Laboratory Accreditation Cooperation (ILAC)"?

What were the considerations for the selection of objects for international comparison, and what is the effect on Japanese industry? Can you explain them in relation to each elemental technology? I think this is synthesis. Also, it is written in the abstract, "We succeeded in linking the national standard of length to the site of production, by powerfully linking the metrological standard and standardization." This seems to be very important in synthesis, but there is no description in the main text about this. Answer (Sonko Osawa)

With the globalization of economy, international Mutual Recognition Agreements in the metrology fields are concluded, and one-stop service is becoming available by presenting the measurement capacity of the national metrology laboratories of the country. In this situation we believe the scenario shown in Fig. 3 provides the three-dimensional coordinate measurement standard to users most effectively.

For the link between standardization and metrological standard, explanations are provided in sections 3.1 and 5.1, and we added the contribution of laser tracker assessment to standardization in section 4.1. 